This item was submitted to Loughborough's Research Repository by the author.

Items in Figshare are protected by copyright, with all rights reserved, unless otherwise indicated.

\title{
Hybrid satellite terrestrial relay networks with cooperative non-orthogonal multiple access
}

PLEASE CITE THE PUBLISHED VERSION

https://doi.org/10.1109/LCOMM.2018.2815610

PUBLISHER

(C) IEEE

VERSION

AM (Accepted Manuscript)

LICENCE

CC BY-NC-ND 4.0

\section{REPOSITORY RECORD}

Yan, Xiaojuan, Hailin Xiao, Kang An, Gan Zheng, and Weiping Tao. 2019. "Hybrid Satellite Terrestrial Relay Networks with Cooperative Non-orthogonal Multiple Access". figshare. https://hdl.handle.net/2134/34466. 


\title{
Hybrid Satellite Terrestrial Relay Networks with Cooperative Non-Orthogonal Multiple Access
}

\author{
Xiaojuan Yan, Hailin Xiao, Kang An, Gan Zheng, and Weiping Tao
}

\begin{abstract}
In this letter, we investigate the outage probability (OP) and ergodic capacity of downlink hybrid satellite terrestrial relay networks (HSTRNs) with a cooperative non-orthogonal multiple access (C-NOMA) scheme, in which a user with better channel condition acts as a relay node and forwards information to other users, thus alleviating the masking effect of users with poor channel conditions in heavy shadowing. Specifically, the exact analytical expression for the $O P$ of the considered system is derived. Furthermore, the ergodic capacity expression is also developed to facilitate the performance evaluation of the proposed framework. Finally, simulations are provided to show the impact of key parameters on the considered system and the superiority of introducing the C-NOMA scheme to the HSTRNs.
\end{abstract}

Index Terms-Hybrid satellite terrestrial relay networks, cooperative non-orthogonal multiple access, outage probability, ergodic capacity.

\section{INTRODUCTION}

$I^{N}$ NCORPORATING the benefits of relaying techniques into satellite systems, hybrid satellite terrestrial relay networks (HSTRNs) can significantly improve the performance of the user whose direct link is unavailable or deteriorated. Until now, several works have been done on the HSTRNs from various key performance measures, such as the outage probability (OP) and ergodic capacity [1]-[4]. Despite of the advantage of the HSTRNs, the time division multiple access (TDMA) scheme adopted in those aforementioned works cannot meet the increasing requirement for high resource efficiency because two time slots are needed to serve a user with a deteriorated link quality. Moreover, the TDMA scheme prefers to serve user with better link qualities to achieve an optimal throughput. Thus, the quality of service for users with deteriorated links may be sacrificed when the number of users is large, which is a common scenario in future satellite communications [3]. Under this condition, other multiple access scheme should be taken into consideration to achieve performance enhancement.

This work was supported by the National Natural Science Foundation of China under Grants 61471392 and 61472094, the UK EPSRC under grant EP/N007840/1, and the Innovation Project of Guangxi Graduate Education.

$\mathrm{X}$. Yan and H. Xiao are with the School of Information and Communication, Guilin University of Electronic Technology, Guilin 541004, China, and X. Yan is also with the Engineering Training Center, Qinzhou University, Qinzhou 535011, China (e-mail: yxj9609@163.com; xhl_xiaohailin@163.com). H. Xiao is the corresponding author.

K. An is with the National University of Defense Technology, Nanjing 210007, China (e-mail: ankang@ nuaa.edu.cn).

G. Zheng is with the Wolfson School of Mechanical, Electrical, and Manufacturing Engineering, Loughborough University, Loughborough LE11 3TU, U.K. (e-mail: g.zheng@ @lboro.ac.uk).

W. Tao is with the College of Electronics and Information Engineering, Qinzhou University, Qinzhou 535011, China (e-mail: twpcoco@163.com).
Recently, a novel multiple access scheme, referred to as cooperative non-orthogonal multiple access (C-NOMA) scheme, has been proposed and studied in a number of works such as [5]-[8]. The key merit of C-NOMA is to transmit multiple signals simultaneously by using the NOMA scheme [9] in the transmission phase, then a user with a better channel condition acts as a relay node and forwards information to other users during the cooperation phase [5]. In this way, more users can gain access than the TDMA scheme to achieve a higher resource utilization efficiency and an improved performance for users with deteriorated link condition. Obviously, this key advantage is quite beneficial for HSTRNs due to the following reasons: 1) The deployment of an extra terrestrial relay node is unnecessary, which is economically and operationally preferred. 2) The cooperation within the group of adjacent users greatly alleviate the poor link gains of users in spot beam edge or in masking effect due to heavy shadowing. However, to the best of our knowledge, existing works on the combination of C-NOMA scheme and HSTRNs have not yet been reported. To fill this gap, this letter studies the performance of C-NOMA based downlink HSTRNs. Specifically, the OP and ergodic capacity expressions for the proposed system are derived. Simulations are provided to show the impact of key parameters on the system performance and the superiority of introducing the C-NOMA scheme to the HSTRNs.

\section{SYSTEM MODEL}

Consider a land mobile satellite communicate system with two pre-paired terrestrial users ${ }^{1}$, User $p$ and User $q$, via the C-NOMA scheme. Each node in the considered system is equipped with a single antenna. We further assume that Users $p$ and $q$ are located in the same spot beam but with different locations. The overall communication takes place in two orthogonal time slots. During the first phase, the satellite broadcasts a superposed signal $x\left(x=\sqrt{\alpha P_{\mathrm{s}}} x_{p}+\sqrt{(1-\alpha) P_{\mathrm{s}}} x_{q}\right)$ to satellite users, the received signal at User $j(j=p, q)$ is

$$
y_{j}=\sqrt{L_{j} G_{s} G_{j}\left(\varphi_{j}\right)} h_{j} x+n_{j},
$$

where $\alpha(0 \leq \alpha \leq 1)$ is a fraction of the transmit power $P_{\mathrm{S}}$ allocated to User $p, x_{j}\left(\mathrm{E}\left[\left|x_{j}\right|^{2}\right]=1\right)$ and $n_{j}\left(\mathrm{E}\left[\left|n_{j}\right|^{2}\right]=\right.$ $N_{0}$ ) are the transmit signal and the additive white Gaussian noise (AWGN) at User $j$, respectively. $L_{j}$ denotes the free space loss (FSL) of the link from the satellite to Users $j$.

\footnotetext{
${ }^{1}$ Only two users are considered here since a NOMA group with two users has been included in the Third Generation Partnership Project (3GPP) [10] and an important conclusion drawn from [11] is that a lower sum rate can be obtained when more than two users are admitted into a cluster.
} 
Here, we consider $L_{p}=L_{q}$ since users within a beam present similar FSL towards the satellite [9]. $G_{s}$ is the antenna gain at the satellite. $G_{j}\left(\varphi_{j}\right)=G_{j}\left(\frac{J_{1}\left(u_{j}\right)}{2 u_{j}}+36 \frac{J_{3}\left(u_{j}\right)}{u_{j}^{3}}\right)^{2}[12]$ is the beam gain depending on both satellite beam pattern and the position of User $j$ with $J(\cdot)$ denoting the Bessel function and $u_{j}=2.07123 \frac{\sin \varphi_{j}}{\sin \varphi_{j 3 \mathrm{~dB}}} . G_{j}$ denotes the antenna gain at User $j, \varphi_{j}$ is the angle between User $j$ and the beam center with respect to the satellite, and $\varphi_{j 3 \mathrm{~dB}}$ denotes the $3-\mathrm{dB}$ angle. As for the fading model, a widely-adopted Shadowed Rician (SR) fading model is adopted and the probability density function (PDF) of $\left|h_{j}\right|^{2}$ is given by [2] as

$$
f_{\left|h_{j}\right|^{2}}(x)=\alpha_{j} e^{-\beta_{j} x}{ }_{1} F_{1}\left(m_{j} ; 1 ; \delta_{j} x\right),
$$

where $\alpha_{j}=0.5\left(2 b_{j} m_{j} /\left(2 b_{j} m_{j}+\Omega_{j}\right)\right)^{m_{j}} / b_{j}, \beta_{j}=0.5 / b_{j}$, $\delta_{j}=0.5 \Omega_{j} / b_{j} /\left(2 b_{j} m_{j}+\Omega_{j}\right), 2 b_{j}$ and $\Omega_{j}$ are the average power of the multipath component and line-of-sight (LoS) component, respectively, $m_{j}\left(m_{j}>0\right)$ denotes the Nakagami$m$ parameter, and ${ }_{1} F_{1}(a ; b ; c)$ represents the confluent hypergeometric function [13, Eq (9.100)]. Moreover, we assume User $p$ has a better channel condition than that of User $q$, i.e., $G_{q}\left(\varphi_{q}\right)\left|h_{q}\right|^{2}<G_{p}\left(\varphi_{p}\right)\left|h_{p}\right|^{2}$ in this letter.

At the receiver side, the user with worse channel condition can decode its own information directly. Thus, the signal-tointerference-plus-noise ratio (SINR) for User $q$ is

$$
\gamma_{q}^{1}=\frac{(1-\alpha) P_{\mathrm{s}} Q_{q}\left|h_{q}\right|^{2}}{\alpha P_{\mathrm{s}} Q_{q}\left|h_{q}\right|^{2}+N_{0}}
$$

where $Q_{q}=L_{q} G_{s} G_{q}\left(\varphi_{q}\right)$. At the same time, the user with better channel condition first decodes the information of User $q$ according to the principle of successive interference cancellation (SIC). So, the decoding SINR can be derived as

$$
\gamma_{p \rightarrow q}=\frac{(1-\alpha) P_{\mathrm{s}} Q_{p}\left|h_{p}\right|^{2}}{\alpha P_{\mathrm{s}} Q_{p}\left|h_{p}\right|^{2}+N_{0}},
$$

where $Q_{p}=L_{p} G_{s} G_{p}\left(\varphi_{p}\right)$. One can obtain that $\gamma_{q}^{1}<\gamma_{p \rightarrow q}$ since $G_{q}\left(\varphi_{q}\right)\left|h_{q}\right|^{2}<G_{p}\left(\varphi_{p}\right)\left|h_{p}\right|^{2}$. Then, User $p$ decodes its own information, and the received SINR at User $p$ is

$$
\gamma_{p}=\alpha P_{\mathrm{s}} Q_{p}\left|h_{p}\right|^{2} / N_{0}
$$

During the second time phase, User $p$ forwards the decoded information to User $q$, and the received SINR at User $q$ is

$$
\gamma_{q}^{2}=P_{p} Q_{p q}\left|h_{p q}\right|^{2} / \delta^{2},
$$

where $Q_{p q}=G_{p} G_{q} / d_{p q}^{u}, P_{p}$ is the transmit power at User $p$, $d_{p q}, h_{p q}$, and $\delta^{2}$ are the distance, the channel coefficient, and the variance of AWGN of User $p \rightarrow$ User $q$, and $u$ is the path loss exponent. In this letter, we model the terrestrial link, $h_{p q}$, as a Nakagami- $m$ fading channel, the PDF of $\left|h_{p q}\right|^{2}$ can be written as

$$
f_{\left|h_{p q}\right|^{2}}(x)=\frac{\xi^{m} x^{m-1}}{\Gamma(m)} e^{-\xi x},
$$

where $\Gamma(\cdot)$ is the Gamma function, $\xi=m / \Omega$ with $m$ being the fading severity parameter and $\Omega$ being the average power.

\section{PERFORMANCE ANALYSIS}

In this section, key performance merits, including OP and ergodic capacity, are derived in the following subsections.

\section{A. Outage probability}

1) OP of User p: Based on the downlink NOMA protocol, an outage of User $p$ can occur when either the decoding SINR $\gamma_{p \rightarrow q}$ falls below a predefined threshold $\gamma_{\text {th } q}$, or the received SINR $\gamma_{p}$ below a threshold $\gamma_{\operatorname{th} p}$ [6], [7], namely,

$$
\begin{aligned}
P_{\text {out } p}\left(\gamma_{\text {th }}\right) & =\operatorname{Pr}\left(\gamma_{p \rightarrow q} \leq \gamma_{\text {th } q}\right)+\operatorname{Pr}\left(\gamma_{p} \leq \gamma_{\text {th } p}, \gamma_{p \rightarrow q}>\gamma_{\text {th }}\right) \\
& =F_{\gamma_{p \rightarrow q}}\left(\gamma_{\text {th } q}\right)+F_{\gamma_{p}}\left(\gamma_{\text {th } p}\right)\left[1-F_{\gamma_{p \rightarrow q}}\left(\gamma_{\text {th } q}\right)\right] .(8)
\end{aligned}
$$

From (4) and (5), the cumulative distribution functions (CDFs) of $F_{\gamma_{p \rightarrow q}}\left(\gamma_{\mathrm{th} q}\right)$ and $F_{\gamma_{p}}\left(\gamma_{\mathrm{th} p}\right)$ can be respectively written as $F_{\gamma_{p \rightarrow q}}\left(\gamma_{\text {th } q}\right)=\operatorname{Pr}\left(P_{\mathrm{s}} Q_{p}\left|h_{p}\right|^{2}\left(1-\alpha-\alpha \gamma_{\text {th } q}\right) / N_{0} \leq \gamma_{\text {th } q}\right)$ and $F_{\gamma_{p}}\left(\gamma_{\mathrm{th} p}\right)=\operatorname{Pr}\left(\alpha P_{\mathrm{s}} Q_{p}\left|h_{p}\right|^{2} / N_{0} \leq \gamma_{\mathrm{th} p}\right)$. Applying the technique in [13, Eqs. (9.14.1), (3.381.1)], we get

$$
\begin{gathered}
F_{\gamma_{p \rightarrow q}}\left(\gamma_{\mathrm{th} q}\right)=\left\{\begin{array}{cc}
\sum_{k=0}^{\infty} \Xi_{p} \gamma\left(k+1, \frac{\gamma_{\mathrm{th} q} \beta_{p} N_{0} / Q_{p}}{P_{\mathrm{s}}\left(1-\alpha-\alpha \gamma_{\mathrm{th} q}\right)}\right), & \frac{1-\alpha}{\alpha}>\gamma_{\mathrm{th} q} \\
1, & \frac{1-\alpha}{\alpha} \leq \gamma_{\mathrm{th} q}
\end{array}\right. \\
F_{\gamma_{p}}\left(\gamma_{\mathrm{th} p}\right)=\sum_{k=0}^{\infty} \Xi_{p} \gamma\left(k+1, \frac{\gamma_{\mathrm{th} p} \beta_{p} N_{0}}{\alpha P_{\mathrm{s}} Q_{p}}\right)
\end{gathered}
$$

where $\Xi_{p}=\frac{\alpha_{p}\left(m_{p}\right)_{k} \delta_{p}^{k}}{(k !)^{2} \beta_{p}^{k+1}}$ with $(a)_{k}=\frac{\Gamma(a+k)}{\Gamma(a)}$ [13] and $\gamma(a, x)=$ $\int_{0}^{x} e^{-t} t^{a-1} d t$ represents the incomplete Gamma function [13, Eq. (8.350.1)]. Substituting (9) and (10) into (8), we can get the OP of User $p$.

2) OP of User q: According to the principle of the decodeand-forward (DF) relaying strategy and the maximal ratio combining (MRC) scheme, the output SINR at User $q$ can be expressed as $\gamma_{q}=\min \left\{\gamma_{p \rightarrow q}, \gamma_{q}^{1}+\gamma_{q}^{2}\right\}$. Thus, the OP of User $q$ can be expressed as

$$
P_{\text {out } q}\left(\gamma_{\text {th } q}\right)=1-\left[1-F_{\gamma_{q}^{1}+\gamma_{q}^{2}}\left(\gamma_{\text {th } q}\right)\right]\left[1-F_{\gamma_{p \rightarrow q}}\left(\gamma_{\text {th } q}\right)\right] \text {. }
$$

Due to the fact that $F_{\gamma_{p \rightarrow q}}\left(\gamma_{\text {thq }}\right)$ in (11) has been obtained by (9), the remaining task is to compute $F_{\gamma_{q}^{1}+\gamma_{q}^{2}}\left(\gamma_{t h q}\right)$, which can be re-expressed as

$$
\begin{gathered}
F_{\gamma_{q}^{1}+\gamma_{q}^{2}}\left(\gamma_{\mathrm{th} q}\right)=\operatorname{Pr}\left(\gamma_{q}^{1} \leq \gamma_{\mathrm{th} q}-\gamma_{q}^{2}, \gamma_{q}^{2} \leq \gamma_{\mathrm{th} q}\right) \\
=\left\{\begin{array}{l}
\underbrace{\int_{0}^{\Delta} F_{\left|h_{q}\right|^{2}}[g(z)] f_{\left|h_{p q}\right|^{2}}(z) d z}_{I_{1}}, \frac{1-\alpha}{\alpha} \geq \gamma_{\mathrm{th} q} \\
\underbrace{\int_{\Delta\left(1-\frac{1-\alpha}{\alpha \gamma_{\mathrm{th} q}}\right)}^{\Delta} F_{\left|h_{q}\right|^{2}}[g(z)] f_{\left|h_{p q}\right|^{2}}(z) d z}_{I_{3}} \underbrace{\int_{0}^{\Delta\left(1-\frac{1-\alpha}{\left.\alpha \gamma_{\mathrm{th} q}\right)} f_{\left|h_{p q}\right|^{2}}(z) d z\right.}, \frac{1-\alpha}{\alpha}<\gamma_{\mathrm{th} q}}_{I_{2}},
\end{array}\right.
\end{gathered}
$$

where $g(z)=\frac{\gamma_{\text {th } q} N_{0}(1-z / \Delta)}{P_{\mathrm{s}} Q_{q}\left(1-\alpha-\alpha \gamma_{\mathrm{th} q}(1-z / \Delta)\right)}$ with $\Delta=\frac{\gamma_{\mathrm{th}} \delta^{2}}{P_{p} Q_{p q}}$. Since PDFs of $\left|h_{q}\right|^{2}$ and $\left|h_{p}\right|^{2}$ have the same form, following similar steps as those in the derivation of $F_{\gamma_{p}}\left(\gamma_{\mathrm{th} p}\right)$, we get

$$
F_{\left|h_{q}\right|^{2}}[g(z)]=\sum_{k=0}^{\infty} \Xi_{q} \gamma\left(k+1, g(z) \beta_{q}\right),
$$




$$
I_{1}=\sum_{k=0}^{\infty} \Xi_{q} \sum_{n=0}^{\infty} \frac{(-1)^{n+r} \beta_{q}^{w} N_{0}^{w} \gamma_{\text {thq }}^{-l}}{n ! w P_{\mathrm{s}}^{w} Q_{q}^{w} \Gamma(m)} \sum_{l=0}^{\infty}\left(\begin{array}{c}
-w \\
l
\end{array}\right) \frac{(1-\alpha)^{l}}{(-\alpha)^{w+l}} \sum_{r=0}^{\infty} \frac{(\xi \Delta)^{m+r}}{r !(m+r)}{ }_{2} F_{1}(l, m+r ; m+r+1 ; 1)
$$

$$
I_{2}=\sum_{k=0}^{\infty} \Xi_{q} \sum_{n=0}^{\infty} \frac{(-1)^{n+r} \beta_{q}^{w} N_{0}^{w} \gamma_{\text {th } q}^{-l}}{n ! w P_{\mathrm{s}}^{w} Q_{q}^{w} \Gamma(m)} \sum_{l=0}^{\infty}\left(\begin{array}{c}
-w \\
l
\end{array}\right) \frac{(1-\alpha)^{l}}{(-\alpha)^{w+l}} \sum_{r=0}^{\infty} \frac{(\xi \Delta)^{m+r}}{r !(m+r)}{ }_{2} F_{1}(l, m+r ; m+r+1 ; 1)\left[1-\left(1-\frac{1-\alpha}{\alpha \gamma_{\mathrm{th} q}}\right)^{r+m}\right] .
$$

where $\Xi_{q}=\frac{\alpha_{q}\left(m_{q}\right)_{k} \delta_{q}^{k}}{(k !)^{2} \beta_{q}^{k+1}}$. By substituting (7), (13), and the expression of $g(z)$ into (12), expanding $\gamma\left(k+1, g(z) \beta_{q}\right)$ and $e^{-\xi x}$ into series representations with [13, Eq. (8.354.1)] and [13, Eq. (1.211.1)], respectively, and utilizing [13, Eq. (3.194.1)], $I_{1}$ defined in (12) can be derived as (14), in which $w=n+k+1$ and ${ }_{2} F_{1}(\cdot)$ represents the hypergeometric functions [13, Eq. (9.100.1)]. Then, based on (14), $I_{2}$ can be derived as (15) by applying the Newton-Leibniz formula $\int_{a}^{b} f(x) d x=F(b)-F(a)$. At last, $I_{3}$ can be derived by inserting (7) into (12) and applying [13, Eq. (3.381.1)], as

$$
I_{3}=\frac{\gamma\left(m, \xi \Delta\left(1-\frac{1-\alpha}{\alpha \gamma_{\mathrm{th} q}}\right)\right)}{\Gamma(m)} .
$$

Substituting (9) and (12)-(16) into (11), we can obtain the OP of User $q$.

The OP of the considered network is defined as the event that neither User $p$ nor User $q$ can detect information reliably [9], i.e., $P_{\text {out }}=1-\left[1-P_{\text {out } p}\left(\gamma_{\text {th }}\right)\right]\left[1-P_{\text {out } q}\left(\gamma_{\text {th } q}\right)\right]$. Substituting (8) and (11) into this equation, we can directly obtain the OP of the proposed system.

\section{B. Ergodic capacity}

The ergodic capacity is defined as the expected value of the instantaneous end-to-end mutual information [3]. In this letter, it can be written as

$$
\mathrm{E}_{\text {erg }}=\mathrm{E}\left[\log \left(1+\gamma_{p}\right)\right]+\mathrm{E}\left[\log \left(1+\gamma_{q}\right)\right] .
$$

Substituting (2) and (5) into (17), we have

$$
\begin{aligned}
& \mathrm{E}\left[\log \left(1+\gamma_{p}\right)\right] \\
& \stackrel{(a)}{=} \frac{1}{\ln 2} \int_{0}^{\infty} G_{2,2}^{1,2}\left[\alpha P_{\mathrm{s}} Q_{p} y / N_{0} \mid \begin{array}{l}
1,1 \\
1,0
\end{array}\right] f(y) d y \\
& \stackrel{(b)}{=} \frac{\alpha_{p} \beta_{p}^{-1}}{\ln 2 \Gamma\left(m_{p}\right)} G_{1,[1: 2], 0,[2: 2]}^{1,1,2,1}\left[\begin{array}{c|c}
\frac{-\delta_{p}}{\beta_{p}} & 1,1 \\
1-m_{p} ; 1,1 \\
-- \\
\frac{\alpha P_{s} Q_{p}}{\beta_{p} N_{0}} & 0,0 ; 1,0
\end{array}\right] \text {, }
\end{aligned}
$$

where $G[\cdot \mid \cdot]$ denotes the Meijer-G functions [13, Eq. (9.301)]. Here, (a) is obtained by expanding $\log (1+x)$ into Meijer-G functions with the aid of [14, Eq. (11)], (b) arises by expanding ${ }_{1} F_{1}(a ; b ; c)$ in $(2)$ into Meijer-G functions according to [13, Eq. (9.34.8)] and applying [15, Eq. (2.6.2)].

According to the output SINR at User $q$, we have

$$
\mathrm{E}\left[\log \left(1+\gamma_{q}\right)\right]=\min \left\{\mathrm{E}\left[\log \left(1+\gamma_{p \rightarrow q}\right), \mathrm{E}\left[\log \left(1+\gamma_{q}^{1}+\gamma_{q}^{2}\right)\right]\right]\right\} .
$$

We define $\Psi_{1}$ and $\Psi_{2}$ to denote the first and second expected values of (19), respectively. Based on (4) and the rules of Logarithm, $\Psi_{1}$ can de further derived as

$\Psi_{1}=\mathrm{E}\left[\log \left(1+\frac{P_{s}}{N_{0}} Q_{p}\left|h_{p}\right|^{2}\right)\right]-\mathrm{E}\left[\log \left(1+\frac{\alpha P_{s}}{N_{0}} Q_{p}\left|h_{p}\right|^{2}\right)\right]$.

Note that the second expected value of (20) has been obtained by (18), the first term of (20) can also be derived by following similar steps. Since $\Psi_{2}$ consists of two different fading distributions and its PDF is mathematically intractable. Here, we seek to consider the approximation expression for $\Psi_{2}$, which can be obtained as [3]

$\Psi_{2} \approx \frac{1}{2 \ln 2}\left[\ln \left(1+\mathrm{E}\left[\gamma_{q}^{\mathrm{mrc}}\right]\right)-\frac{\mathrm{E}\left[\left(\gamma_{q}^{\mathrm{mrc}}\right)^{2}\right]-\left(\mathrm{E}\left[\gamma_{q}^{\mathrm{mrc}}\right]\right)^{2}}{2\left(1+\mathrm{E}\left[\gamma_{q}^{\mathrm{mrc}}\right]\right)^{2}}\right]$

where $\gamma_{q}^{\mathrm{mrc}}=\gamma_{q}^{1}+\gamma_{q}^{2}$. Due to the independence of $\left|h_{q}\right|^{2}$ and $\left|h_{p q}\right|^{2}$, we have $\mathrm{E}\left[\gamma_{q}^{\mathrm{mrc}}\right]=\mathrm{E}\left[\gamma_{q}^{1}\right]+\mathrm{E}\left[\gamma_{q}^{2}\right]$ and $\mathrm{E}\left[\left(\gamma_{q}^{\mathrm{mrc}}\right)^{2}\right]=$ $\mathrm{E}\left[\left(\gamma_{q}^{1}\right)^{2}\right]+\mathrm{E}\left[\left(\gamma_{q}^{2}\right)^{2}\right]+2 \mathrm{E}\left[\gamma_{q}^{1}\right] \mathrm{E}\left[\gamma_{q}^{2}\right]$. Firstly, we compute the $\eta$-order moments $(\eta=1,2)$ of $\gamma_{q}^{1}$, as

$$
\mathrm{E}\left[\left(\gamma_{q}^{1}\right)^{\eta}\right]=\int_{0}^{\infty} \frac{(1-\alpha)^{\eta} P_{\mathrm{s}}^{\eta} Q_{q}^{\eta} y^{\eta}}{\left(\alpha P_{\mathrm{s}} Q_{q} y+N_{0}\right)^{\eta}} f(y) d y .
$$

Expressing ${ }_{1} F_{1}\left(m_{q}, 1, \delta_{q} y\right)=\sum_{k=0}^{\infty} \frac{\left(m_{q}\right)_{k} \delta_{q}^{k}}{(k !)^{2}} y^{k}$ according to [13, Eq. (9.100.1)] and utilizing [16, Eq. (2.3.6.9)], we can get

$$
\begin{aligned}
\mathrm{E}\left[\left(\gamma_{q}^{1}\right)^{\eta}\right] & =\alpha_{q} \sum_{k=0}^{\infty} \frac{(1-\alpha)^{\eta}\left(m_{q}\right)_{k} \delta_{q}^{k}}{(k !)^{2}\left(\alpha P_{\mathrm{s}} Q_{q} N_{0}^{-1}\right)^{1+k}} \Gamma(k+\eta+1) \\
& \times \Psi\left(k+\eta+1, k+2 ; \frac{\beta_{q} N_{0}}{\alpha Q_{q} P_{\mathrm{s}}}\right),
\end{aligned}
$$

where $\Psi(\cdot)$ is the confluent Hypergeometric function [13, Eq. (9.210.2)]. Then, substituting (7) into (6) along with [13, Eq. (3.381.4)], the $\eta$-order moments of $\gamma_{q}^{2}$ can be derived as

$$
\mathrm{E}\left[\left(\gamma_{q}^{2}\right)^{\eta}\right]=\frac{P_{p}^{\eta} G_{p q}^{\eta} \Gamma(m+\eta)}{\Gamma(m) \xi^{\eta} \delta^{2 \eta}} .
$$

Thus, (21) can be obtained via (23) and (24) with $\eta=1,2$.

At last, by substituting (18) and (21) into (17), the ergodic capacity of the considered system can be obtained.

\section{NUMERICAL RESULTS}

This section provides numerical results to corroborate our theoretical results and show the superiority of introducing the C-NOMA scheme to the HSTRNs. Here, we set the 


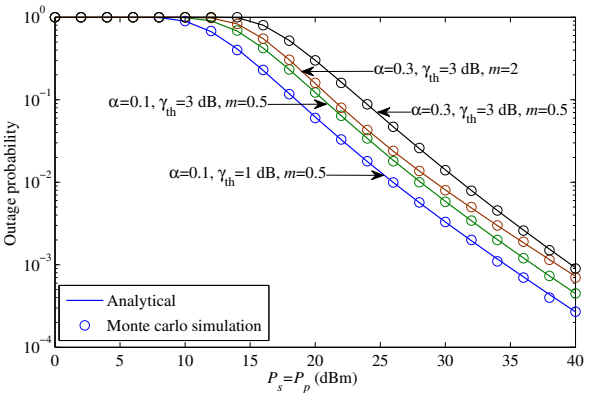

Fig. 1. The $\mathrm{OP}$ of the considered system versus $P_{\mathrm{s}}=P_{p}$ with various values of $\alpha, \gamma_{\mathrm{th}}$, and $m$.

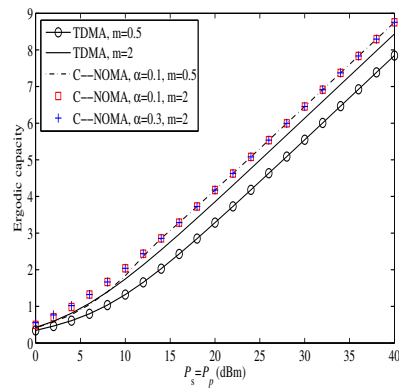

(a)

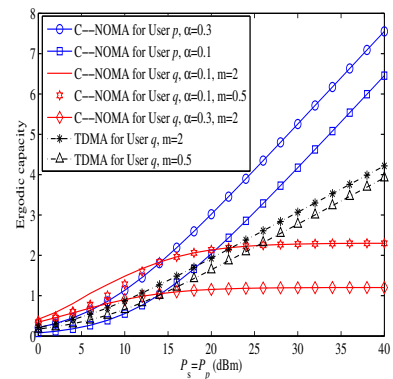

(b)
Fig. 2. Ergodic capacity versus $P_{\mathrm{S}}=P_{p}$ with different values of $\alpha$ and $m$ : (a) the sum capacity and (b) each user.

carried frequency to be $2 \mathrm{GHz}, G_{s}=52.1 \mathrm{dBi}, \varphi_{p}=0.1^{\circ}$, $\varphi_{q}=0.8^{\circ}, \varphi_{p 3 \mathrm{~dB}}=\varphi_{q 3 \mathrm{~dB}}=0.3^{\circ}, u=2, d_{p q}=1 \mathrm{Km}$, $\gamma_{\text {th } p}=\gamma_{\text {th } q}=\gamma_{\text {th }}, \Omega=1$, and $G_{p}=G_{q}=3.5 \mathrm{dBi}$. Moreover, we assume that User $p$ undergoes an infrequent light shadowing (ILS) with $\left(m_{p}, b_{p}, \Omega_{p}\right)=(19.4,0.158,1.29)$, while User $q$ experiences a frequent heavy shadowing (FHS) with $\left(m_{q}, b_{q}, \Omega_{q}\right)=\left(0.739,0.063,8.97 \times 10^{-4}\right)$ [2], [12].

We first conduct numerical simulations to show the impact of various parameters on the OP performance of the considered network, as depicted in Fig. 1. It can be seen that the OP performance degrades when either the outage threshold $\gamma_{\text {th }}$ increases, or the power allocation factor $\alpha$ increases, or the fading severity parameter $m$ decreases. The reason behind is that an outage of the considered system happens when any user can not achieve a reliable detection, while the OP of User $p$ given in (8) or that of User $q$ given in (11) degrades as $\alpha$ or $\gamma_{\text {th }}$ increases. Meanwhile, a more favourable condition is achieved when $m$ is larger. Moreover, we can see that the analytical results agree well with the Monte Carlo simulations.

Then, we analyze ergodic capacity of two schemes. The ergodic sum capacity with different parameters is firstly studied in Fig. 2(a), from which we can see that the capacity curves with the C-NOMA scheme outperform those with the TDMA scheme. That is because in two time slots, multiple users can access in the C-NOMA scheme, while only one user whose direct link is deteriorated and helped by a DF relay node can be served with the TDMA scheme. Moreover, we note that the values of $\alpha$ and $m$ have little effect on the proposed system performance. This phenomenon can be explained by the fact that the $\gamma_{q}$ always equals to $\gamma_{p \rightarrow q}$ when $\gamma_{p \rightarrow q} \leq \gamma_{q}^{1}+\gamma_{q}^{2}$.
Thus, the ergodic sum capacity of the proposed system can be derived as $\mathrm{E}_{e r g}=\mathrm{E}\left[\log \left(1+P_{s} Q_{p}\left|h_{p}\right|^{2} / N_{0}\right)\right]$. Furthermore, the ergodic capacities of each user are depicted in Fig. 2(b). As illustrated, capacity curves of User $q$ with the C-NOMA scheme are first superior and then at some point inferior to those with the TDMA scheme. The point occurs at a lower transmit power for a larger $\alpha$. This implies that with a suitable $\alpha$, the performance of the user with deteriorated channel quality can be further improved with the C-NOMA scheme when the transmit power is not high. It can also be observed from Fig. 2(b) that the more power allocated to User $p$, i.e., $\alpha$ gets larger, the better the ergodic capacity of User $p$ is, but the worse the performance of User $q$ is.

\section{CONCLUSIONS}

In this letter, we have investigated the performance of downlink HSTRNs with the C-NOMA scheme. In particular, we have derived the exact expression for the OP of the considered system. Then, the ergodic capacity has also been analyzed. Simulation results have been provided to show the effect of different channel parameters, the power allocation factor, and the threshold on the system performance. Our findings suggest the superiority of intrducing the C-NOMA scheme in downlink HSTRNs.

\section{REFERENCES}

[1] L. Yang and M. O. Hasna, "Performance analysis of amplify-and-forward hybrid satellite-terrestrial networks with cochannel interference," IEEE Trans. Commun., vol. 63, no. 12, pp. 5052-5061, Dec. 2015.

[2] K. An et al., "Performance analysis of multi-antenna hybrid satellite terrestrial relay networks in the presence of interference," IEEE Trans. Commun., vol. 63, no. 11, pp. 4390-4404, Nov. 2015.

[3] K. An, M. Lin, and T. Liang, "On the performance of multiuser hybrid satellite terrestrial relay networks with opportunistic scheduling," IEEE Commun. Lett., vol. 19, no. 10, pp. 1722-1725, Oct. 2015.

[4] P. K. Upadhyay and P. K. Sharma, "Max-max user-relay selection scheme in multiuser and multirelay hybrid satellite-terrestrial relay systems," IEEE Commun. Lett., vol. 20, no. 2, pp. 268-271, Feb. 2016.

[5] Z. Ding et al., "Cooperative non-orthogonal multiple access in $5 \mathrm{G}$ systems," IEEE Commun. Lett., vol. 19, no. 8, pp. 1462-1465, Aug. 2015.

[6] X. Yue, Y. Liu, A. Nallanathan, and Z. Ding, "Exploiting full/half-duplex user relaying in NOMA systems," IEEE Trans. Commun., in press.

[7] Y. Liu et al., "Cooperative non-orthogonal multiple access with simultaneous wireless information and power transfer," IEEE J. Sel. Areas Commun., vol. 34, no. 4, pp. 938-953, Apr. 2016.

[8] Z. Zhang, Z. Ma, M. Xiao, Z. Ding, and P. Fan, "Full-duplex deviceto-device-aided cooperative nonorthogonal multiple access," IEEE Trans. Veh. Technol., vol. 66, no. 5, pp. 4467-4471, May 2017.

[9] M. Caus et al., "NOMA and interference limited satellite scenarios." in Proc. IEEE ACSSC'16, Pacific Grove, CA, USA, Mar. 2017, pp. 497-501.

[10] Z. Yang, Z. Ding, P. Fan, and Z. Ma, "Outage performance for dynamic power allocation in hybrid non-orthogonal multiple Aaccess systems," IEEE Commun. Lett., vol. 20, no. 8, pp. 1695-1698, Aug. 2016.

[11] M. Zeng et al., "Capacity comparison between MIMO-NOMA and MIMO-OMA with multiple users in a cluster," IEEE J. Sel. Areas Commun., vol. 35, no. 10, pp. 2413-2424, Oct. 2017.

[12] G. Zheng, S. Chatzinotas and B. Ottersten, "Generic optimization of linear precoding in multibeam satellite systems," IEEE Trans. Wireless Commun., vol. 11, no. 6, pp. 2308-2320, June 2012.

[13] I. S. Gradshteyn and I. M. Ryzhik, Table of Integrals, Series, and Products, 7th ed. New York, NY, USA: Academic, 2007.

[14] V. S. Adamchik and O. I. Marichev, "The algorithm for calculating integrals of hypergeometric type functions and its realization in reduce systems," in Proc. ISSAC'90, Tokyo, Japan, Aug. 1990, pp. 212-224.

[15] A. M. Mathai and R. K. Saxena, The H-function with applications in statistics and other disciplines, New York, NY, USA:Wiley, 1978.

[16] A. P. Prudnikov, Y. A. Brychkov, and O. I. Marichev, Integrals and Series, Vol. 1, 4th ed. Taylor and Francis, 2002. 